\title{
Erfolgreiche Dritte Gemeinsame Jahrestagung von SETAC-GLB und GDCh-Fachgruppe „Umweltchemie und Ökotoxikologie“
}

\author{
Motto „Neue Problemstoffe in der Umwelt - Erfassung, Wirkungen, Lösungen“ \\ 23.-26. September 2008 in Frankfurt am Main
}

\author{
Matthias Oetken $\cdot$ Wilhelm Püttmann $\cdot$ Dominic Kaiser $\cdot$ Julia Regnery $\cdot$ Jörg Oehlmann
}

Erhalten: 14. November 2008/Akzeptiert: 12. Dezember 2008/Online veröffentlicht: 10. Januar 2009

(C) Springer-Verlag 2009

Mit dem Verhalten und den Wirkungen von Chemikalien in der Umwelt sowie mit Lösungsansätzen für damit verbundene Umweltprobleme befasste sich vom 23. bis 26. September 2008 in Frankfurt am Main die dritte gemeinsame Tagung des deutschsprachigen Zweigs der Society of Environmental Toxicology and Chemistry (SETAC GLB) und der Fachgruppe Umweltchemie und Ökotoxikologie der Gesellschaft Deutscher Chemiker (GDCh). Bereits in den Jahren 2002 und 2004 fanden gemeinsame Jahrestagungen beider Gesellschaften in Braunschweig bzw. in Aachen statt.

Das übergeordnete Motto der diesjährigen Tagung lautete „Neue Problemstoffe in der Umwelt - Erfassung, Wirkungen und Lösungsmöglichkeiten“. Im Fokus standen beispielsweise Pharmaka, perfluorierte Chemikalien, Pestizide und Nanopartikel. Als „Neue Problemstoffe“ werden Stoffe betrachtet, die entweder erst seit kurzer Zeit in die Umwelt eingetragen werden oder aber bereits seit Dekaden in der Umwelt auftreten und erst in jüngerer Zeit als problematisch wahrgenommen werden. Die 300 Teilnehmer aus beiden Gesellschaften demonstrierten auf eindrucksvolle Weise, wie wichtig dieses Thema ist.

In drei parallelen Vortragssitzungen wurden folgende 16 Themenbereiche behandelt:

- Monitoring von Chemikalien in der Umwelt

- Effektmodellierung

\footnotetext{
M. Oetken $(\bowtie) \cdot$ D. Kaiser $\cdot$ J. Oehlmann

Institut für Ökologie, Evolution und Diversität, Abteilung Aquatische Ökotoxikologie, Goethe-Universität Frankfurt/Main,

Siesmayerstr. 70, 60323 Frankfurt am Main, Deutschland

E-Mail: oetken@bio.uni-frankfurt.de

W. Püttmann $\cdot$ J. Regnery

Institut für Atmosphäre und Umwelt, Abteilung Umweltanalytik,

Goethe-Universität Frankfurt/Main,

Altenhöferallee 1, 60438 Frankfurt am Main, Deutschland
}

- Biozide/Pflanzenschutzmittel: Wirkung und Abbau

- Pharmazeutika

- Tools zur Wirkungserfassung

- Umweltanalytik

- Biologisches Effektmonitoring

- Verteilung zwischen verschiedenen Umweltkompartimenten

- Technische Lösungen

- Effekte auf Lebensgemeinschaften/Biodiversität

- Risikobewertung und regulatorische Aspekte

- Sozial-ökologische Lösungsansätze

- Integrative Expositionserfassung

- Ökotoxizität von Mischungen

- Polyfluorierte Substanzen und

- Sekundäres organisches Aerosol

Das breite Themenspektrum wurde in insgesamt 90 Vorträgen und 89 Postern beleuchtet und diskutiert. Die Beitragenden kamen aus Universitäten und Forschungseinrichtungen sowie aus der Industrie und aus Behörden. Erfreulich war die große Beteiligung von Nachwuchswissenschaftlern. Die Poster waren während der gesamten Tagung ausgestellt und konnten im Rahmen von fünf Postersessions ausgiebig diskutiert werden.

Der Eröffnungsvortrag von Dr. Steffi Richter vom Bundesministerium für Umwelt, Naturschutz und Reaktorsicherheit widmete sich dem Thema: ,SAICM: Der strategische Ansatz für ein internationales Chemikalienmanagement im internationalen und nationalen Kontext". Auf der ersten internationalen Chemikalienkonferenz 2006 in Dubai wurde SAICM basierend auf dem Freiwilligkeitsprinzip als globales Instrument zur Minimierung der negativen Auswirkungen von Chemikalien bis 2020 eingerichtet. Dabei sollen weltweit vorhandene und teilweise konkurrierende Aktivitäten zur Chemikaliensicherheit übergreifend gebündelt, Lücken im Chemikalienmanagement identifiziert und 
geschlossen sowie die Kluft zwischen Industrie- und Entwicklungsländern im Umgang mit Chemikalien verringert werden. Drei sich ergänzende Dokumente konkretisieren die Inhalte. Allein der globale Maßnahmenplan zum Chemikalienmanagement empfiehlt als eine Art Leitfaden mehr als 270 Maßnahmen auf 36 Themengebieten. Wie Frau Dr. Richter in ihrem Vortrag betonte, stehen bei der nationalen Umsetzung von SAICM insbesondere die Schwellenund Entwicklungsländer im Vordergrund, da die in der EU vorgeschlagenen Maßnahmen bereits weitgehend durch nationale und EU-Regelungen flankiert sind. Es sei sogar so, dass beispielsweise die in der EU-REACH-Verordnung zugrunde liegende Sicherheitsphilosophie über SAICM weiter verbreitet und besser genutzt werden kann. Im Mai 2009 findet in Genf die zweite internationale Chemikalienkonferenz statt. Dort soll unter anderem bilanziert werden, ob sich SAICM bislang bewährt hat.

Basis politischer Weichenstellungen und Entscheidungen müssen plausible wissenschaftliche Ergebnisse sein, wenngleich diese gerade im Umweltbereich nicht immer übereinstimmend interpretiert werden. So fällt es aufgrund benutzerfreundlicher Methoden immer leichter, immer mehr, immer neue Chemikalien in der Umwelt zu erfassen. Gerade wegen der sich stetig verbessernden Analysenmethoden fragte Prof. Dr. Walter Giger von der Eidgenössischen Anstalt für Wasserversorgung, Abwasserreinigung und Gewässerschutz (EAWAG) im schweizerischen Dübendorf im Rahmen des ersten Plenarvortrags zum Themenfeld Erfassung: „Was kommt als Nächstes?“ Zwar sind viele Stoffe, deren Umweltproblematik in den letzten Jahrzehnten nachgewiesen wurde, heute zuminderst in Europa weitgehend reguliert (z.B. Nonylphenolethoxylate), aber es kommen andere Stoffe hinzu, wobei insbesondere die Stoffpalette der sog. ,personal care products“ in Zukunft einer besonderen Beachtung bezüglich negativer Auswirkungen für die aquatische Umwelt bedarf. Dabei gilt es, deren Einträge in die aquatische Umwelt sowie deren Verhalten bei der Abwasserreinigung und der Uferfiltration zu ermitteln. Herr Giger machte anhand mehrerer Beispiele deutlich, dass ein in der Umweltanalytik zunehmend eingesetztes Analysenverfahren, bestehend aus einer Kopplung von Flüssigkeitschromatografie und Tandem-Massenspektrometrie uns heute den Blick auf „neue“ umweltrelevante Substanzen und Substanzklassen eröffnet, die zum Teil schon lange in die aquatische Umwelt eingetragen werden, aber in Ermangelung adäquater Analysentechniken lange Zeit nicht detektiert wurden. Am Abend des ersten Tages erwartete die Konferenzteilnehmer im Foyer des Casinogebäudes des ehemaligen IG-Farben-Hauses der gemütliche Teil der Tagung, das Get together, das der international erfahrene Konzertpianist Georg Klemp am Flügel eröffnete. Zu späterer Stunde gab es Musik vom Band, zu der im Festsaal ausgelassen getanzt wurde.
Am folgenden Konferenztag thematisierte Prof. Dr. Thomas Braunbeck (Universität Heidelberg) im zweiten Plenarvortrag die Wirkung neuer Problemstoffe auf Organismen und stellte fest, dass sich die Belastung von Organismen in der Umwelt von einem durch Unfälle und kurzfristige Kontaminationen mit z.T. sehr hohen Schadstoffkonzentrationen geprägten Szenario zu einer kontinuierlichen Exposition gegenüber einer Vielzahl von Substanzen in relativ niedrigen Konzentrationen verschoben hat. Das Instrumentarium zum Nachweis biologischer Effekte hinke der Entwicklung chemisch-analytischer Methoden meist hinterher. „Neuartige Effekte“ gingen beispielsweise von hormonwirksamen Substanzen oder von Pharmaka aus, also von Substanzen, die vom Menschen explizit für eine spezifische Wirkung entwickelt wurden und eingesetzt werden. Unverständlicherweise werde für einzelne Substanzgruppen regelrecht versäumt, ökotoxikologische Kenndaten, die über akut toxische Wirkungen hinausgehen, zu ermitteln. Als Beispiel nannte Braunbeck perfluorierte Tenside (insbesondere Perfluoroctansulfonsäure - PFOS, Perfluoroctansäure - PFOA), die aufgrund ihrer Persistenz und ihres Bioakkumulationspotenzials in Europa z.T. bereits reguliert werden, über deren chronische Wirkungen bisher aber kaum Informationen existieren. Bei Nanopartikeln sei die Ökotoxikologie von echten Erkenntnissen hinsichtlich des langfristigen Gefährdungspotenzials noch weit entfernt, so Braunbeck. In der Ökotoxikologie geht der Trend hin zu Methoden der molekularen Zell- und Entwicklungsbiologie. Genomics, Transcriptomics und Proteomics sind Techniken, mit denen große Erwartungen verbunden sind. Jedoch werden Spezifität und Empfindlichkeit noch kritisch betrachtet. Daher treten etablierte Ansätze wieder in den Vordergrund, zu denen beispielsweise klassische histologische Techniken gehören.

Nicht minder wichtig erscheint auch das letzte Themenfeld im Untertitel des Tagungsmottos „Lösungen“, die sich nach der Erfassung und Wirkungsanalyse von bestimmten Stoffen oder Stoffgruppen als besondere Herausforderung darstellen. Dieser Thematik widmete sich Prof. Dr. Hansruedi Siegrist (EAWAG, Dübendorf, $\mathrm{CH}$ ) im abschließenden dritten Plenarvortrag am dritten Tagungstag. Der Schwerpunkt dieses Übersichtsvortrags lag auf den technischen Lösungsmöglichkeiten wie z. B. der Einführung eines Umweltlabels für Arzneimittel, die separate Behandlung von stark kontaminierten Abwässern, beispielsweise aus Krankenhäusern, oder auch die Urinseparation. Die Zudosierung von Ozon oder Pulveraktivkohle in den Ablauf der biologischen Klärstufe sind weitere technische Möglichkeiten zur Reduktion des Spurenstoffeintrags in die Umwelt. Gegenwärtig werden Versuche durchgeführt, um die technische Machbarkeit der Ozonung als Bestandteil der Kläranlagentechnik zu prüfen. Neben der Frage nach den Kosten und dem Energieverbrauch bedarf es insbesondere einer 
Klärung der Frage, ob durch die Ozonung aus den primären Fremdstoffen Oxidationsprodukte (Metabolite) gebildet werden, die evtl. toxisch wirken können. Am Nachmittag fanden drei Exkursionen statt - eine erste Exkursion führte die Teilnehmer zum Hauptklärwerk der Stadt Wiesbaden, wobei neue Abwasserbehandlungstechniken präsentiert wurden. Im Verlaufe der zweiten angebotenen Exkursion gewannen die Teilnehmer Impressionen im historischen Palmenhaus und dem Tropicarium im Palmengarten Frankfurt. Das Senckenbergmuseum, eines der großen Naturkundemuseen in Deutschland, war Ziel der dritten Exkursion - hier konnte die heutige Vielfalt des Lebens, die Entwicklung der Lebewesen sowie die Verwandlung unserer Erde über Jahrmillionen hinweg bestaunt werden. Abgerundet wurde der Tag durch das traditionelle Tagungsbankett, das diesmal im Herzen Frankfurts, im Schirn-Cafe stattfand. Dort erwartete die Konferenzteilnehmer ein Sektempfang sowie ein üppiges Buffet. Im Laufe des Abends wurde für gute Stimmung durch DJ Sportswagon gesorgt und bis weit in die Nacht ausgiebig getanzt.

Ein wichtiges Ziel von SETAC-GLB ist die Förderung des wissenschaftlichen Nachwuchses. In diesem Jahr wurden drei Nachwuchswissenschaftler prämiert, die ihre Arbeiten zu Beginn des zweiten Konferenztages dem Publikum vorstellten. Dr. Oliver Körner (RIFCon GmbH, Hirschberg) erhielt den Preis für die beste Dissertation („,Field and laboratory approaches to assess estrogen disruption in the brown trout Salmo trutta"). Den Preis für die beste Diplomarbeit erhielten zwei Absolventen der RWTH Aachen, Silvana Siehoff („Interaktionen von Daphnia magna und Periphyton“) und Hanno Zielke (,Vergleich verschiedener Methoden zur Sedimentextraktion im Fischeitest mit dem Zebrabärbling (Danio rerio)").

In der Abschlussveranstaltung prämierten GDCh und SETAC gemeinsam jeweils die besten drei Vorträge und Poster mit einem Geldpreis. Die Auswahl der Preisträger erfolgte nicht durch eine spezielle Jury; vielmehr stimmten die Tagungsteilnehmer selbst mittels eines Bewertungsbogens über die Preisvergabe ab. Bei den Vorträgen freuten sich die folgenden Preisträger: 1. Kirsten Wendler (Schriesmann), 2. Lutz Ahrens (Geesthacht), 3. Kerstin Ulrich (Heidelberg) und bei den Postern: 1. László Dören (Schweinsberg), 2. Ulrike Morgner (Leipzig), 3. und 4. Jonas Baumann (Frankfurt am Main). Sowohl SETAC-GLB als auch die GDCh-Fachgruppe Umweltchemie und Ökotoxikologie vergeben auch im nächsten Jahr Förderpreise, sodass schon jetzt dazu aufgerufen wird, hierfür herausragende Diplom-, Doktor- oder sonstige wissenschaftliche Arbeiten einzureichen. Details hierzu werden in Kürze auf den jeweiligen Homepages verfügbar sein (www.setac-glb.org bzw._www.umweltchemiegdch.de).

Durch das große Engagement der Sektionsvorsitzenden, der Mitglieder des Wissenschaftlichen Komitees (Christine Achten (Universität Wien, A), Klaus-Peter Ebke (Mesocosm GmbH, Homberg/Ohm), Elke Fries (Universität Osnabrück), Tobias Frische (Umweltbundesamt Dessau), Bettina Hitzfeld (Bundesamt für Umwelt BAFU, Ittigen, CH), Henner Hollert (RWTH Aachen), Udo Hommen (IME Schmallenberg), Adriano Joss (EAWAG, Zürich, CH), Florian Keil (ISOE Frankfurt/Main), Gerhard Lammel (MPI für Chemie, Mainz, D; Masaryk Universität, Brno, CZ), Matthias Liess (UFZ Leipzig, Halle), Werner Manz (BFG, Koblenz), Matthias Oetken (Universität Frankfurt/Main), Wilhelm Püttmann (Universität Frankfurt/Main), Bettina Rechenberg (Umweltbundesamt Dessau), Andreas Schäffer (RWTH Aachen), Peter Seel (HLUG, Wiesbaden), Lennart Weltje (BASF SE, Limburgerhof)) und die vielen helfenden Hände des Organisationskomitees war die Tagung in Frankfurt ein voller Erfolg.

In diesem Jahr ist es den beiden wissenschaftlichen Gesellschaften, SETAC-GLB und GDCh-Fachgruppe „Umweltchemie und Ökotoxikologie" erneut gelungen, ein gemeinsames, ihnen wichtiges Thema zu definieren und somit Kräfte zu bündeln. Der Reiz einer gemeinsamen Tagung liegt gerade in der Interdisziplinarität. Ein Novum war sicherlich nicht nur die Einbeziehung technischer Verfahren zur Reduktion von Problemstoffen in der Umwelt, sondern auch die Diskussion von Strategien zur Entwicklung eines nachhaltigen Problembewusstseins im Umgang mit Chemikalien bei der Bevölkerung. Die erfolgreiche gemeinsame Jahrestagung in Frankfurt am Main macht Mut, dass auch in Zukunft beide Gesellschaften ihre Jahrestagungen zusammen stattfinden lassen - die Vierte Gemeinsame Jahrestagung ist im Jahr 2010 in Dessau geplant.

Weitere Informationen zur Tagung sind unter http://www. setac-glb.de/GLB-Tagungen-96-08.266.0.html zu finden.

Danksagung Unterstützt wurde die Dritte Gemeinsame Jahrestagung der SETAC-GLB und der GDCh-Fachgruppe Umweltchemie und Ökotoxikologie durch die folgenden Institutionen, ohne deren Hilfe die Tagung nicht in dieser Form hätte durchgeführt werden können: BASF SE, Dr. Knoell Consult GmbH, ECT Oekotoxikologie GmbH, F. Hoffmann-La Roche Ltd., Henkel KgaA, IBACON GmbH, LGC Standards, Novartis Pharma AG, RLP AgroScience GmbH, SCC Scientific Consulting Company $\mathrm{GmbH}$, Syngenta Agro $\mathrm{GmbH}$, ThermoFischer Scientific, Umweltbundesamt, Verband der Chemischen Industrie e.V. (VCI), Vereinigung von Freunden und Förderern der Johann Wolfgang Goethe-Universität. 